\title{
Immunocytochemical Localization of Tubulin and Microtubule-Associated Protein 2 During the Development of Hippocampal Neurons in Culture
}

\author{
Alfredo Cáceres, ${ }^{*}, 1$ Gary A. Banker, ${ }^{*}$ and Lester Binder $\dagger$ \\ *Department of Anatomy, Neil Hellman Medical Research Building, Albany Medical College, Albany, New York \\ 12208, and †Department of Biology, University of Virginia, Charlottesville, Virginia 22908
}

In dissociated-cell cultures prepared from the embryonic rat hippocampus, neurons establish both axons and dendrites, which differ in geometry, in ultrastructure, and in synaptic polarity. We have used immunocytochemistry with monoclonal antibodies to study the regional distribution of beta-tubulin and microtubule-associated protein 2 (MAP2) in hippocampal cultures and their localization during early stages of axonal and dendritic development.

After development for a week or more in culture, when axons and dendrites were well-differentiated, the distribution of these two proteins was quite different. Beta-tubulin was present throughout the nerve cell, in soma, dendrites, and axon. It was also present in all classes of non-neuronal cells, astrocytes, fibroblasts, and a presumptive glial progenitor cell. In contrast, MAP2 was preferentially localized to nerve cells; within neurons, MAP2 was present in soma and dendrites, but little or no immunostaining was detectable in axons.

Both beta-tubulin and MAP2 were present in nerve cells at the time of plating. From the earliest stages of process extension, beta-tubulin was present in all neuronal processes, both axons and dendrites. Surprisingly, MAP2 was also initially present in both axons and dendrites, extending as far as the axonal growth cone. With subsequent development, MAP2 staining was selectively lost from the axon so that after 1 week in vitro little or no axonal staining remained.

Taken together with earlier results (Cáceres et al., 1984a), these data indicate that the establishment of neuronal polarity, as manifested by the molecular differentiation of the axonal and dendritic cytoskeleton, occurs largely under endogenous control, even under culture conditions in which cell interactions are greatly restricted. MAP2 does not appear to be involved in the determination of neuronal polarity; processes that initially contain MAP2 may become either axons or dendrites.

Among the earliest and most fundamental events in neuronal differentiation is the establishment of the two distinct classes of neuronal processes, axons and dendrites. This event, which is usually associated with the development of an asymmetric and spatially complex morphology, is of critical significance because it establishes a neuron's functional polarity. Moreover, it forms

\footnotetext{
Received Mar. 1, 1985; revised June 4, 1985; accepted June 6, 1985.

This research was supported by NIH Grant NS 17112. Dr. Cáceres' participation in this project was supported in part by a Fogarty International Fellowship (FSTW02910A) and by NIH Grant NS12333 to Dr. O. Steward. We wish to thank Ann Lohr for her excellent technical assistance, Anne Rafferty for her patient help with the photography, and especially Dr. Oswald Steward, whose advice and encouragement were invaluable.

Correspondence should be addressed to Dr. Gary Banker.

' Permanent address: Instituto de Investigacion Médica Mercedes y Martin Ferreyra, Cordoba, Argentina.

Copyright (C) 1986 Society for Neuroscience 0270-6474/86/030714-09\$02.00/0
}

the structural basis for the establishment of molecularly distinct domains within the neuronal membrane, which gives each class of neurons its distinct physiological properties.

Several lines of evidence indicate that the various components of the neuronal cytoskeleton are crucial for the differentiation, elaboration, and maintenance of axons and dendrites (for reviews, see Lasek et al., 1983; Solomon, 1981; Solomon and Zurn, 1981). In addition, recent biochemical and immunocytochemical studies have established that there are molecular differences between the microtubules (Cáceres et al., 1984b; Matus et al., 1981; Vallee, 1982) and neurofilaments (Hirokawa et al., 1984; Shaw et al., 1981) in different regions of a neuron, and in different classes of neurons (Goldstein et al., 1983). Some of the regional differences in microtubules occur early in neuronal development (Bernhardt and Matus, 1982). These results support the notion that the development of the high degree of anisotropy characteristic of neurons may arise from molecular asymmetries of the cytoskeleton. In this regard, microtubules are apt to be especially important because they arise early in neuronal development, usually much before neurofilaments (Pachter and Liem, 1984; Peters and Vaughn, 1967; Shaw et al., 1985), and because they have been implicated in a variety of cellular events, including the outgrowth of neuronal processes and the transport of materials within axons and dendrites.

Although all cellular microtubules are thought to contain alpha- and beta-tubulin as well as microtubule-associated proteins (MAPs), there is a considerable microheterogeneity and compartmentalization of microtubule components in neurons. Different classes of microtubules appear to contain different isotypes of the tubulin subunits (Gozes and Barnstable, 1982; Gozes and Sweadner, 1981; Moura Neto et al., 1983), and these different microtubules appear to be compartmentalized within neurons (Cumming et al., 1983). MAPs are also differentially distributed in neurons. For example, immunocytochemical studies show that MAP2, one of the two classes of high-molecular-weight MAPs (HMW-MAPs), is preferentially associated with dendritic microtubules (Cáceres et al., 1984b; DeCamilli et al., 1984; Huber and Matus, 1984a). This is consistent with its preferential distribution in gray matter, as has been shown biochemically (Vallee, 1982). By contrast, MAP1 appears to be present in both axons and dendrites (Bloom et al., 1984; Huber and Matus, 1984b). Among the lower molecular weight MAPs, there is recent evidence that the "tau" proteins are also differentially distributed (Binder et al., 1984b; Tytell et al., 1984).

It is possible that the synthesis and compartmentation of MAPs may serve as a signal for regulating or even directing cellular events in neurogenesis, such as the differentiation of axons and dendrites. Bernhardt and Matus (1982) have shown that HMW-MAPs are present during the initial phases of dendritic development in cerebellar Purkinje cells. They proposed that the local presence of HMW-MAPs may provide a stimulus 
for the initiation of dendritic outgrowth from the soma and for the continuing extension of dendritic growth cones. In a pheochromocytoma cell line (PC12), neurite outgrowth induced by NGF is accompanied by a selective induction of MAPI (Greene et al., 1983). These authors hypothesize that MAP1 might enhance the formation and stabilization of microtubules and consequently promote neurite outgrowth. Other studies have shown that HMW-MAPs appear only in differentiated neuroblastoma cells (Izant and MacIntosh, 1980; Olmsted and Lyon, 1981) and that the biochemical composition of the MAPs changes profoundly during brain development (Binder et al., 1984a; Burgoyne and Cumming, 1984; Francon et al., 1982; Mareck et al., 1980). Taken collectively these results suggest an important role for MAPs in neuronal development, but because of the different model systems and experimental approaches used, a clear picture of the function MAPs play has not yet emerged.

As part of a research project ultimately directed to determine the mechanisms by which axons and dendrites are formed and acquire their distinct shapes and functional properties, we have chosen to study the possible role of MAPs, using as a model system cell cultures prepared from the hippocampus. Unlike continuous nerve cell lines, hippocampal pyramidal cells in primary culture reproduce many of the characteristic structural features that distinguish them in situ. Most important, they establish two distinct classes of processes that can be identified as axons and dendrites based on their geometry, their ultrastructural features, and their synaptic polarity (Banker and Cowan, 1979; Bartlett and Banker, 1984a, b). We have recently shown that the axons and dendrites that form in culture also differ in the molecular composition of their cytoskeletons. MAP2 is restricted to the soma and dendritic processes, as it is in the hippocampus in situ (Cáceres et al., 1984a). In contrast, the $\mathrm{H}$ subunit of neurofilaments $(200 \mathrm{kDa})$ is present in some of the axons that develop in culture, as shown by immunocytochemistry, but is undetectable in the dendrites (Shaw et al., 1985).

The general advantages of cell culture systems for the study of cytoskeletal organization are well known, but two features of this hippocampal culture system make it especially suitable for studies of the neuronal cytoskeleton in development. First, all phases of development can be observed and studied, from the earliest stages of axonal and dendritic initiation. Second, individual cells can be seen in their entirety, so that regional differences in the cytoskeleton can be directly examined at the single cell level. In the present study, we have examined the distribution of MAP2 in these cultured cells and compared it with the distribution of tubulin, using immunocytochemistry with monoclonal antibodies. We have studied its distribution in well-differentiated neurons and non-neuronal cells; in addition, we have examined the distribution of MAP2 throughout the course of axonal and dendritic development, from the earliest phase of process outgrowth.

\section{Materials and Methods}

\section{Preparation of cell cultures}

Hippocampal neurons were prepared from the brains of 17-20-d-old rat fetuses, as described in detail by Banker and Cowan (1977) and Bartlett and Banker (1984a). Hippocampi were treated with trypsin $\left(0.25 \%\right.$ for $15 \mathrm{~min}$ at $\left.37^{\circ} \mathrm{C}\right)$, washed with $\mathrm{Ca}$ - and $\mathrm{Mg}$-free Hank's balanced solution, and dissociated by repeated passage through a constricted Pasteur pipette. The nerve cell suspensions were then plated onto polylysine-treated $16 \mathrm{~mm}$ coverslips at densities ranging from $5000-15,000$ cells $/ \mathrm{cm}^{2}$. After plating, the cells were incubated for 2-3 hr in Eagle's Minimum Essential Medium (MEM) containing 10\% horse serum to enable the neurons to attach to the substrate.

The coverslips, with neurons attached, were transferred to dishes containing already confluent astroglial cultures (Booher and Sensenbrenner, 1972) and oriented with the nerve cells "down," facing the glial monolayer. It has previously been shown that co-culture of hip- pocampal neurons with astroglial cells greatly enhances neuronal survival (Banker, 1980). The cultures were maintained in MEM supplemented with the N2 mixture of Bottenstein and Sato (1979) plus $0.1 \%$ ovalbumin. Once a week, one-third of the culture medium was replaced with fresh medium.

\section{Production and characterization of monoclonal antibodies}

A detailed description of the preparation and characterization of the monoclonal antibodies used in this study has been presented previously (Cáceres et al., 1984b; Payne, 1982). The antibodies were prepared against microtubules purified from Chinese hamster brain and were obtained as culture supernatants; the anti-tubulin antibodies were purified by protein $\mathrm{A}$ affinity chromatography. Three clones reacting with beta-tubulin (Tu9b, $0.32 \mathrm{mg} / \mathrm{ml}$; Tu12, $0.37 \mathrm{mg} / \mathrm{ml}$; Tu27, $0.28 \mathrm{mg}$ / $\mathrm{ml}$ ) and two reacting with MAP2 (AP9, $0.39 \mathrm{mg} / \mathrm{ml}$; AP13, $0.40 \mathrm{mg} /$ $\mathrm{ml}$ ) were used in this study. The binding specificity of each clone was examined by solid-phase-enzyme-linked immunoassay, and cross-reactivity to other proteins was tested by reacting the antibodies with electroblots of rat hippocampus or cerebellum.

\section{Immunocytochemical procedures}

Cultures were prepared for immunocytochemistry using several procedures: (1) Fixation for $30 \mathrm{~min}$ with warmed $4 \%$ paraformaldchydc plus $0.25 \%$ glutaraldehyde in phosphate buffer, $\mathrm{pH} 7.2$, followed by dehydration in graded ethanols and permeabilization for $30 \mathrm{~min}$ with $95 \%$ ethanol; (2) fixation for $10 \mathrm{~min}$ in methanol at $-20^{\circ} \mathrm{C}$; (3) extraction with detergent to prepare "cytoskeletons" according to the procedure of Schliwa and VanBlerkom (1981), followed by fixation as in method 1 or 2 . In the latter case, the coverslips were washed in microtubulestabilizing buffer $\left(0.13 \mathrm{M}\right.$ HEPES, pH $6.9,2 \mathrm{~mm} \mathrm{MgCl}_{2}, 10 \mathrm{~mm}$ EGTA) for $30 \mathrm{sec}$, then extracted with $0.2 \%$ Triton $X-100$ in the same buffer for $2 \mathrm{~min}$. In the case of the very young cultures, the extraction procedure was performed only for $60 \mathrm{sec}$, since longer incubation with the detergent resulted in considerable cellular destruction. After being washed free of fixative, the cultures were preincubated in $3 \%$ normal rabbit serum or $5 \%$ BSA in PBS for $1 \mathrm{hr}$. The cultures were then incubated for $2-3 \mathrm{hr}$ at $37^{\circ} \mathrm{C}$ with the monoclonal antibodies at dilutions ranging from $1: 15$ to $1: 100$. After washing the cultures for $15 \mathrm{~min}$ in PBS, they were incubated in rabbit anti-mouse IgG $(1: 40)$ followed by peroxidase-antiperoxidase (Sternberger-Meyer, 1:80) and reacted with diaminobenzidine as previously described (Cáceres et al., 1984a, b). Cultures treated identically except for omission of the primary antibody or pretreated with an excess of purified antigen served as controls.

\section{Results}

\section{Tubulin and MAP2 in mature cultures}

The light-microscopic appearance of neurons in dissociated-cell cultures from the hippocampus has been described in detail previously (Banker and Cowan, 1977, 1979; Bartlett and Banker, $1984 a, b)$. In cultures that have been maintained for $1-5$ weeks in vitro, the distinctive appearance of axons and dendrites is readily apparent by LM. These features are well illustrated in Figure $1 A$, which shows a culture after $17 \mathrm{~d}$ in vitro. The dendrites are thick at the base, taper with increasing distance from the cell body, branch in $\mathrm{Y}$-shaped angles, and are relatively short (roughly $100-250 \mu \mathrm{m}$ long at this stage of development). EM has shown that these processes contain ribosomes, exhibit spines, and are exclusively postsynaptic (Bartlett and Banker, 1984b). Axonal processes are long, thin, and quite uniform in diameter. They intersect one another, fasciculate, and form an extensive plexus, so that individual axons cannot be distinguished from small fascicles by LM and axons cannot be followed to their cells of origin. At the electron-microscopic level, the axons lack ribosomes and are exclusively presynaptic (Bartlett and Banker, 1984b). Dendritic segments are also relatively straight, whereas axons frequently follow a tortuous course, even forming small loops.

Figure $1 B$ illustrates the localization of beta-tubulin in this same culture. As reported previously (Cáceres et al., 1984a), immunoreactive beta-tubulin was present in all portions of the 

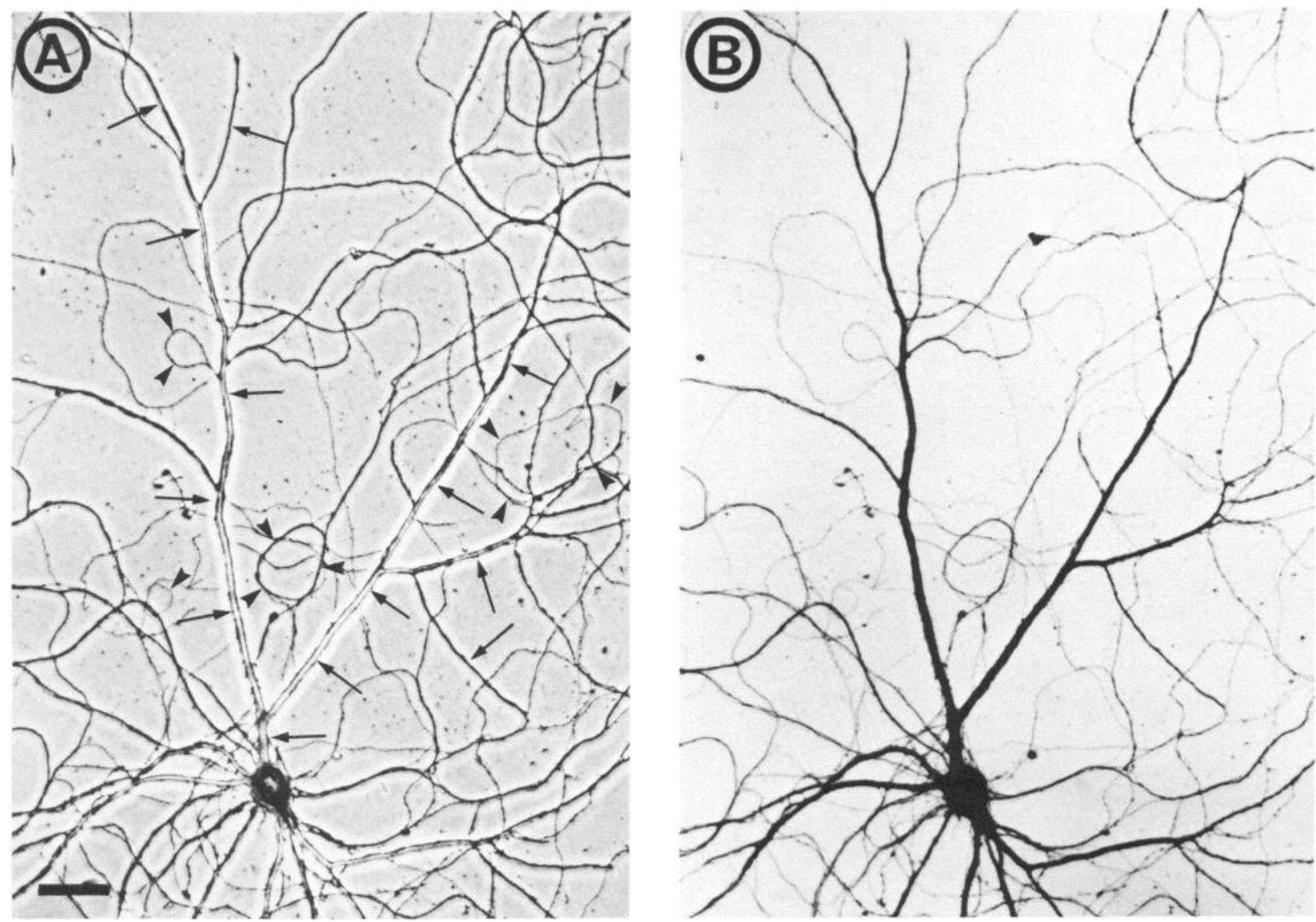

Figure 1. Nerve cell from a hippocampal culture maintained $17 \mathrm{~d}$ in vitro. Several of the processes that arise from the cell body can be traced for a considerable distance and bear the hallmarks of dendrites. The longest of this cell's dendrites is indicated by the arrows $(A)$. The dendrites are thick at the base and taper with distance from the cell body. This decrease in diameter is especially obvious at branch points, the daughter branches being always thinner in diameter than the parent. In several instances these dendritic branches can be traced to their ends. The fine processes present throughout the field are axons. They frequently take a tortuous course, sometimes even forming closed loops (arrowheads, $A$ ). Electron microscopy of similar cultures shows that these background processes consist of small bundles of axons as well as of individual axons. Some axons also run along the surface of the dendrites, although this is not visible by light microscopy. The distribution of beta-tubulin, as revealed following immunocytochemistry with peroxidase-antiperoxidase, is shown in the bright-field micrograph $(B)$. Staining for tubulin appears to be uniform throughout all portions of the dendrites and axons, even the smallest axons being visible. Methanol fixation, staining with clone Tu9B. Bar, 30 $\mu \mathrm{m} ; \times 305$.

neuron-in the perikaryon, throughout all portions of the dendrites that could be followed from the cell body, and in even the thinnest of the axons. Every process that could be seen by phase-contrast microscopy also displayed immunoreactivity for beta-tubulin. It has been shown by immunofluorescence microscopy that alpha-tubulin is also present in all of the neuronal processes that develop from hippocampal neurons in culture (Shaw et al., 1985).

The localization of MAP2 was strikingly different (Fig. 2; see also Cáceres et al., 1984a, Fig. 1). Both perikarya and dendrites were intensely immunoreactive for MAP2, but little or no reactivity was observed in any of the axonal processes. The staining observed in somata and dendrites represents MAP2 that remains associated with the cytoskeleton after extraction with Triton X-100 (Cáceres et al., 1984a). The paucity of axonal MAP2 immunoreactivity could, in principle, result from a modification of the molecule or an alteration in its association with other axonal constituents, but in light of biochemical evidence that MAP2 is much more concentrated in gray matter than in white matter (Vallee, 1982), it seems probable that it represents a difference in the amount of MAP2 in axons and dendrites in culture as well. This restricted localization of MAP2 was observed after development in vitro for times ranging from 7 to $35 \mathrm{~d}$. Identical results were obtained with both MAP2-specific monoclonal antibodies.
The architecture of the dendritic trees of cells in culture, as revealed following staining with anti-MAP2 antibodies, is illustrated in more detail in Figure 3. The considerable extent of higher-order dendritic branching that develops in culture and the consistent decrease in diameter that occurs at branch points, an invariant property of dendrites in situ (Hillman, 1979), are clearly revealed. Even very thin dendritic branches were stained with anti-MAP2, and when compared with phase contrast images, the staining could frequently be traced completely to the end of a dendritic process.

The non-neuronal cells present in these cultures, which have been identified using cell-type specific markers (A. Hodson and G. Banker, unpublished observations), include astrocytes, fibroblasts, and presumptive glial progenitor cells similar to those described by Raff et al. (1983) in cultures of optic nerve. All were stained with antibodies specific for tubulin, but little immunoreactive MAP2 was detected in any of these classes of non-neuronal cells. Hence, MAP2 appears to be preferentially expressed by neuronal cells in hippocampal cultures, just as it is in situ (Cáceres et al., 1984b; Huber and Matus, 1984b).

\section{Distribution of tubulin and MAP2 early in neuronal development}

Immunocytochemical studies using polyclonal antibodies that react with both MAP1 and MAP2 indicate that the HMW- 

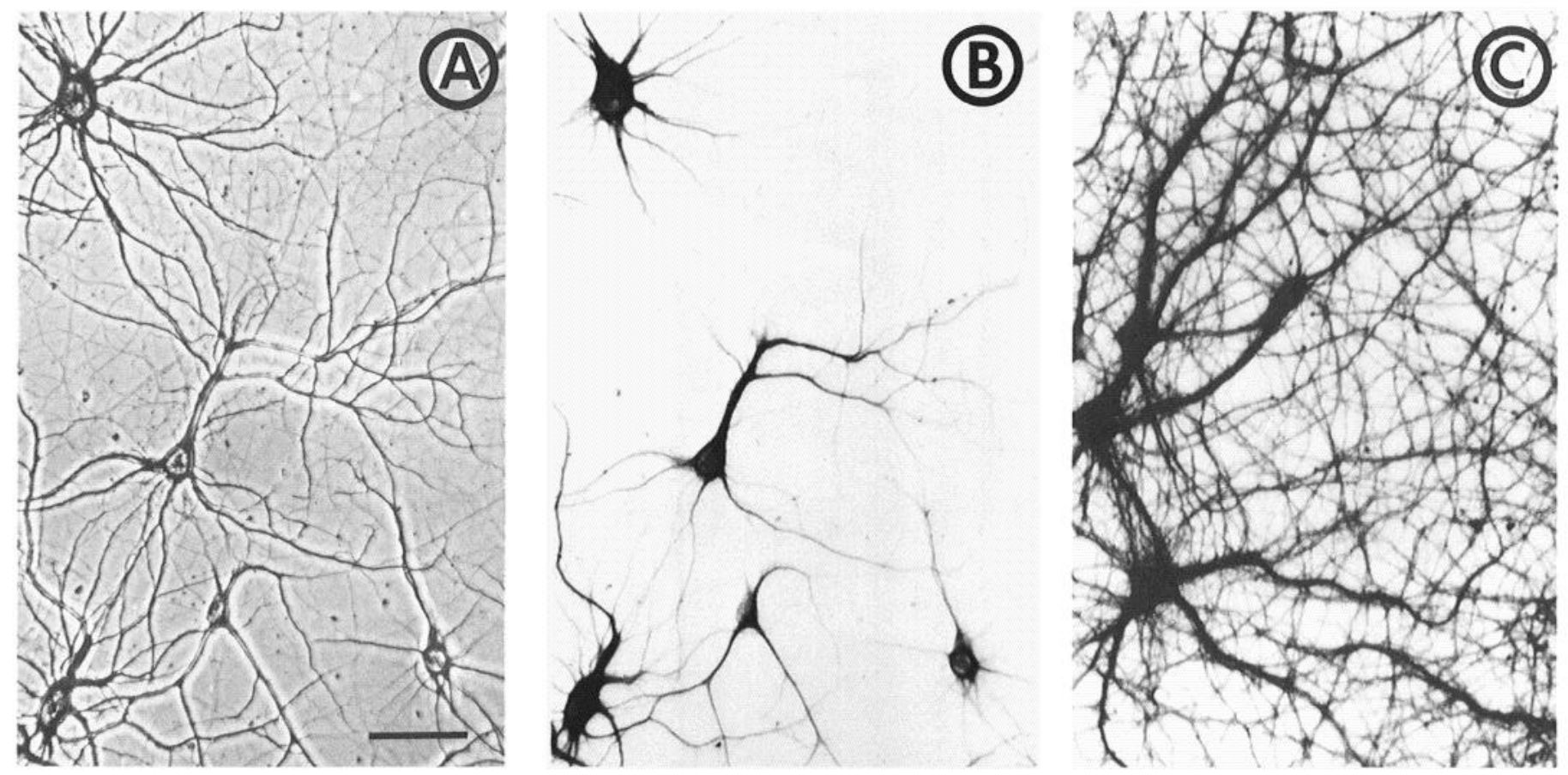

Figure 2. Distribution of MAP2 and beta-tubulin in hippocampal cultures. The phase-contrast micrograph $(A)$ reveals cell bodies and dendrites within a dense network of axonal processes; the bright-field micrograph $(B)$, illustrating MAP2 immunoreactivity, shows intense staining of cell bodies and dendrites, but no apparent staining of axons. In a sister culture reacted with antibody against beta-tubulin $(C)$, all of the neuronal processes are stained. These $15 \mathrm{~d}$ cultures were fixed in cold methanol, then incubated with anti-MAP2 antibody AP9 or anti-tubulin antibody Tu27. Bar, $50 \mu \mathrm{m} ; \times 500$.

MAPs are present in cerebellar Purkinje cells as early as $3 \mathrm{~d}$ after birth (Bernhardt and Matus, 1982). In order to determine if MAP2 is expressed by hippocampal neurons prenatally, at the embryonic stages used to prepare neurons for culture (as early as 17 d gestation), we examined cultures $30 \mathrm{~min}$ after plating, as soon as the cells had adhered to the substrate. At this time the great majority of cells are without processes; only an occasional cell retains the stub of a process that was present before dissociation. Nearly all cells reacted with antibodies against both MAP2 and beta-tubulin (data not shown). Reaction product was detected in the perikarya as well as in those few processes that were present. The presence of these proteins so soon after plating almost certainly indicates that they were present in situ, and hence that MAP2 appears very early in neuronal development.

Within a day after plating, most neurons had begun to extend processes, as shown in Figure 4. Typically these included one major process, which at this stage was $50-100 \mu \mathrm{m}$ in length, and several, much shorter, minor processes (see Banker and Cowan, 1977). The minor processes ranged from flattened lamellipodia to well-defined processes about $20 \mu \mathrm{m}$ long. Sequential photography of individual cells during the first week in culture has shown that the axonal arborization arises by continued growth of the major process while many of the minor processes persist to become the definitive dendrites (Banker, unpublished observations).

We first examined the distribution of beta-tubulin in such cells. Bernhardt and Matus (1982) have reported that, in the case of cerebellar Purkinje cells, tubulin immunoreactivity cannot be detected at early stages of dendritic outgrowth. In hippocampal neurons in culture, immunoreactivity for beta-tubulin appeared to be uniformly distributed throughout the cell (Fig. $4 B$ ). All of the processes, both axons and dendrites, were from the outset heavily stained following exposure to antibodies against beta-tubulin. This staining was not attributable solely to unpolymerized tubulin. Immunofluorescence observations clearly revealed fibrillar staining in the flattened regions from which the dendrites arise, and tubulin immunoreactivity was retained in the developing dendrites following extraction with Triton $\mathrm{X}-100$ under conditions that remove unpolymerized cytoskeletal subunits but preserve microtubules. Preliminary electronmicroscopic observations of hippocampal neurons at this stage show that longitudinally aligned microtubules form the predominant cytoskeletal element in both developing axons and dendrites (Bartlett and Banker, unpublished observations.)

We next examined the localization of MAP2 to determine if it was present in axonal or dendritic processes when they first developed and if it was differentially distributed. Examples of hippocampal neurons stained for MAP2 after 1 or $2 \mathrm{~d}$ in culture are shown in Figure 5. Surprisingly, immunoreactivity for MAP2 was present in all of the developing processes, including the developing axon. This was also true following culture for $2 \mathrm{~d}$, when MAP2 immunoreactivity could be followed to the tips of axons as far as $150 \mu \mathrm{m}$ from the cell bodies. In flattened regions of the cell, the MAP2 staining could sometimes be seen to have a filamentous character. For example, in the neuron shown in Figure $5 D$, the MAP2 appears to be associated with filamentous structures that form a ring within the body of the growth cone. Rings of microtubules have been observed in the growth cones of sensory neurons in culture (Tsui et al., 1983).

\section{Loss of MAP2 during axonal development}

The loss of MAP2 immunoreactivity in older axons could come about in one of two ways. There might simply be turnover of the MAP2 initially present, together with a preferential routing of newly synthesized MAP2 to the dendrites. Alternatively the short, MAP2-containing axons present at 1 or $2 \mathrm{~d}$ in vitro could be resorbed as other, MAP2-free axons developed. Sequential observation of individual cells has established that retraction of some axonal processes does occur during this stage of axonal development, although many of the initial axons do persist (Banker, unpublished observations). 


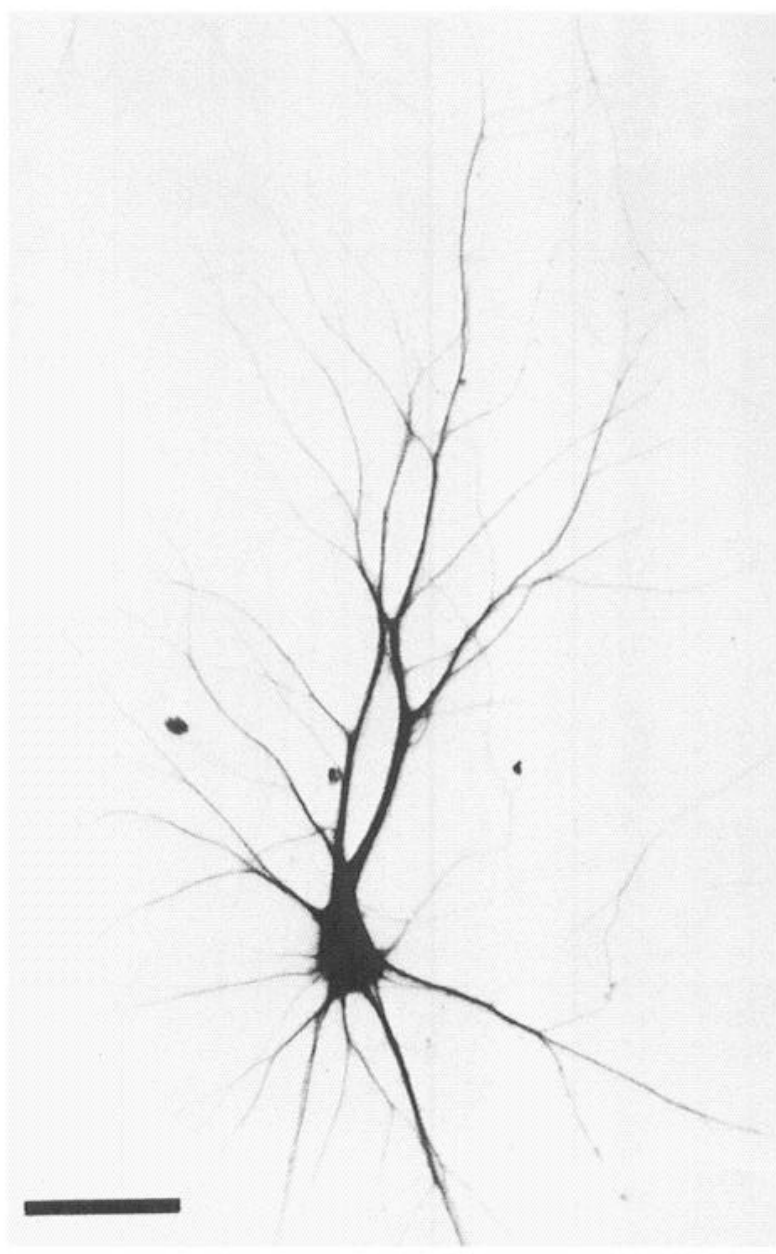

Figure 3. Hippocampal neuron stained with antibody against MAP2 to show the extent of its dendritic tree. The considerable degree of higher-order branching that develops in such cultures is clearly revealed by such immunostaining. In its overall size and in its pattern, the dendritic tree of this cell bears a striking resemblance to that of hippocampal pyramidal neurons in situ. This culture, which had been maintained for $14 \mathrm{~d}$, was extracted with Triton X-100 (0.2\%) for $2 \mathrm{~min}$ before fixation and staining with monoclonal antibody AP9. A slight staining of some axons is seen in preparations treated with Triton, but it is difficult to determine if this is significantly above background. Phase-contrast micrographs of this cell (not illustrated) show the density of axons surrounding this cell to be comparable to that illustrated in Figure 1. Bar, $50 \mu \mathrm{m} ; \times 325$.

Observations of the distribution of MAP2 during this transition period provided direct evidence indicating that MAP2 is lost by turnover or redistribution. When 3 or $4 \mathrm{~d}$ cultures were examined after fixation with low concentrations of glutaraldehyde together with formaldehyde, MAP2 immunoreactivity appeared to be preferentially localized in the distal portions of the axonal arborization, as well as throughout the dendrites. In some cases, axonal MAP2 was restricted to the growth cone and the segment of axon immediately proximal to it (Fig. 6). In cultures at a comparable stage that were fixed with methanol or with formaldehyde alone, little or no axonal immunoreactivity for MAP2 could be detected. These observations could be explained if the axonal MAP2 loses its association with polymerized cytoskeletal elements but persists for a short period of time in a soluble form. If new MAP2-free microtubules are assembled at the base of the axon during elongation, as other studies suggest (Lasek, 1982), the residual MAP2 would become concentrated in the distal axon. By about the end of the first week in culture,
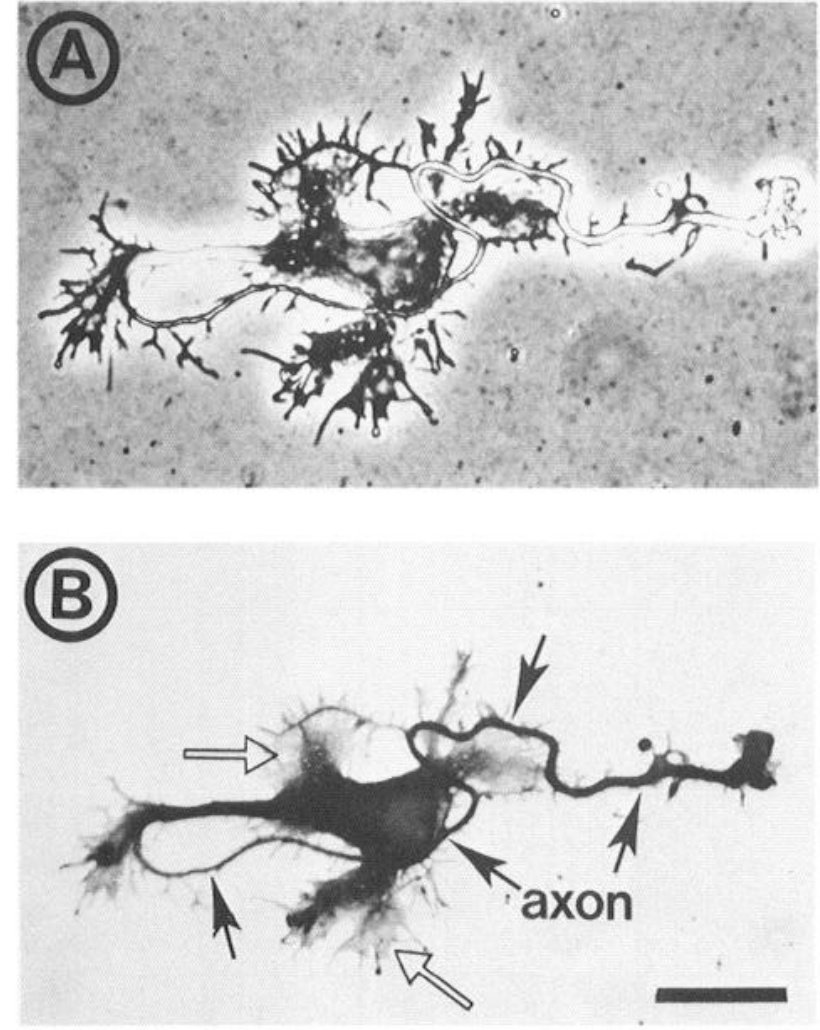

Figure 4. Hippocampal neuron after $1 \mathrm{~d}$ in culture stained with antibodies against beta-tubulin. The axon $(B$, arrows) originates from the left side of the soma, then bends back on itself and follows a tortuous course to the right. This cell has not yet developed definitive dendritic processes, but several lamellipodia-like structures ( $B$, open arrows) extend from the cell body. The bright-field micrograph $(B)$ reveals apparently uniform staining of all portions of the cell, including the lamellipodial extensions. This culture was fixed in formaldehydeglutaraldehyde $(4 \%-0.2 \%)$, then treated with monoclonal antibody Tu9B. Bar, $25 \mu \mathrm{m} ; \times 525$.

axonal MAP2 staining had largely disappeared. Throughout this period of development, immunoreactivity for beta-tubulin appeared uniformly distributed in both the axonal and dendritic processes.

It may also be noted in Figure 6 that MAP2 immunoreactivity appears to be present at the base of the axon (arrowheads). Previous light- and electron-microscopic studies have shown that the axons in these cultures most typically arise as branches from the proximal portion of a dendrite, rather than directly from the cell soma (Bartlett and Banker, 1984a). Thus, this immunoreactivity probably represents staining of the dendritic process from which the axon originates. Immunoreactivity for MAP2 at the origin of the axon was also seen following formaldehyde or methanol fixation and persisted at later developmental stages, after MAP2 staining of the distal axon had disappeared.

\section{Discussion}

The focus of this report concerns the localization of MAP2 and tubulin at early stages of neuronal development in culture. Our observations show that the compartmentalization of MAP2 immunoreactivity occurs in an unexpected way: MAP2 is not selectively excluded from the axon at early stages of axonal outgrowth, but rather is selectively lost from the axon following an initial period when its distribution is uniform throughout the cell. In addition, our results show that MAP2 appears very early in neuronal differentiation and that its subsequent compart- 

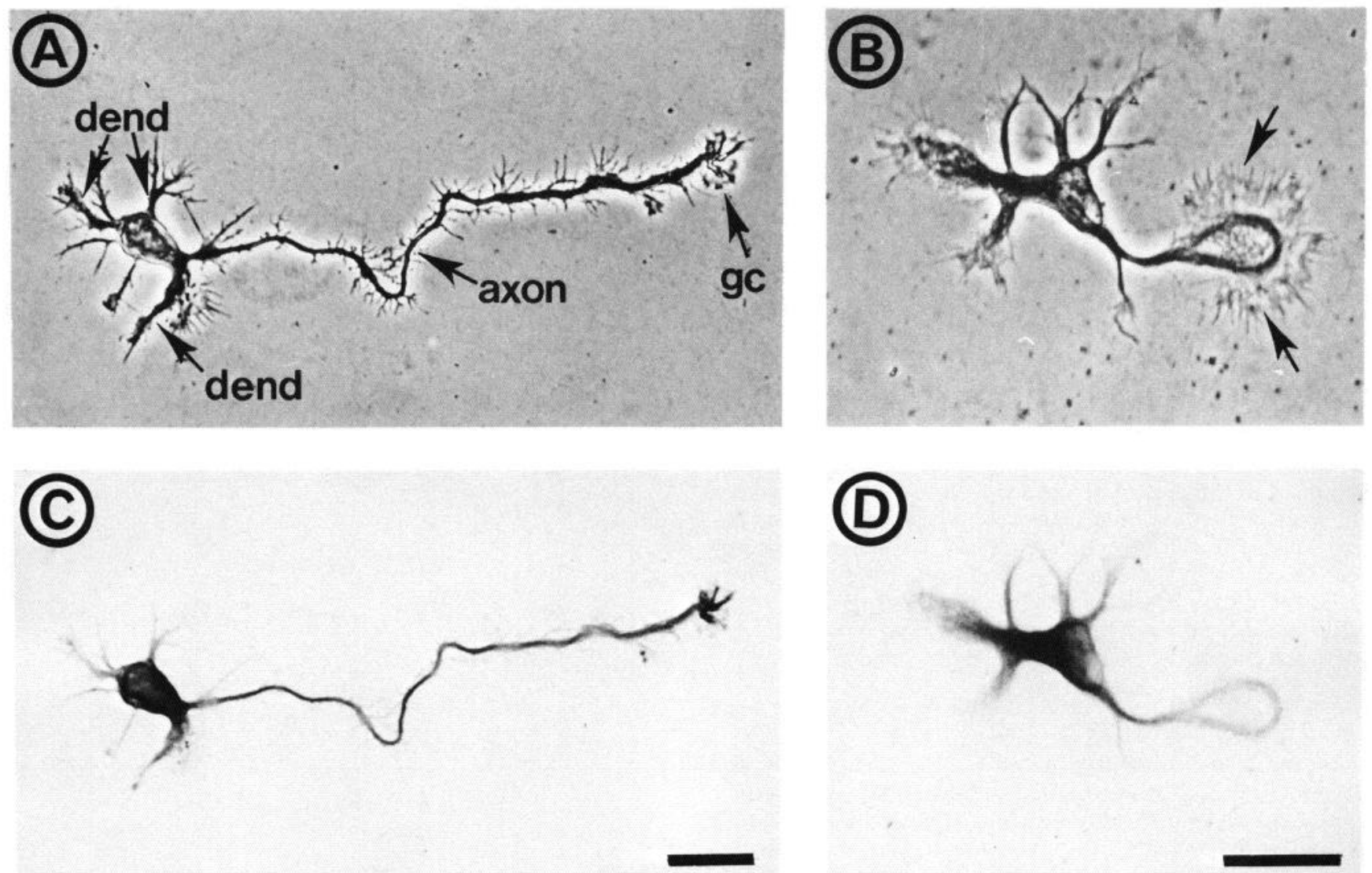

Figure 5. Hippocampal neurons after 1 or $2 \mathrm{~d}$ in culture stained with antibodies against MAP2. The cell illustrated by phase-contrast microscopy in $A$ has a single axon and several short presumptive dendrites (dend). MAP2 staining, seen in the bright-field micrograph $(C)$, is present along the full length of the axon as far as the base of its growth cone $(g c)$. In some instances, as in $D$, the staining in flattened areas of the cell has a somewhat fibrillar character. In the large growth cone that originates from this cell ( $B$, arrows), the MAP2-containing fibrils form a loop. Both cultures were fixed in cold methanol after growth for $1(A$ and $C)$ or $2(B$ and $D)$ d; they were stained with clones AP9 and AP13, respectively. Bar, $20 \mu$ m; $A, C$, $\times 565 ; B, D, \times 785$.

mentalization is largely under endogenous control, independent of cell-cell interactions.

The distribution of MAP2 in situ has not yet been studied at comparably early stages. Therefore, before discussing the significance of these observations, it is important to emphasize that the distribution of MAP2 and tubulin in older hippocampal cultures parallels their distribution in situ (Cáceres et al., 1984a, and this report). In well-differentiated hippocampal neurons in culture we observed that MAP2 was preferentially localized to the cell bodies and dendrites; little or no labeling of axons or of non-neuronal cells was seen. Beta-tubulin appears to be uniformly distributed in the axonal and dendritic processes of hippocampal neurons and is present as well in all of the classes of non-neuronal cells found in these cultures. These observations concerning the localization of MAP2 and tubulin in mature hippocampal cultures are in all respects consistent with studies of the distribution of MAP2 and tubulin in situ. Thus, they demonstrate that the compartmentalization of MAP2 occurs appropriately in hippocampal cultures and, hence, that these cultures provide a suitable model system for analyzing the role of MAP2 in dendritic development.

\section{The appearance of MAP2 early in neuronal development}

Biochemical studies have shown that MAP2 is present in the rat cerebellum, telencephalon, and thalamus on postnatal Day 10, the earliest stage yet examined (Binder et al., 1984a; Burgoyne and Cumming, 1984). Immunocytochemical studies us- ing a polyclonal antibody that recognizes both MAP1 and MAP2 have detected these proteins in cerebellar Purkinje cells on postnatal Day 3, roughly $10 \mathrm{~d}$ after Purkinje cell generation; earlier stages were not examined (Bernhardt and Matus, 1982). Our results show that MAP2 is present in hippocampal neurons at considerably earlier stages of development than have been studied previously. We detected both MAP2 and tubulin in freshly dissociated hippocampal neurons prepared from 18 and $19 \mathrm{~d}$ embryos. The majority of neurons in the hippocampus at this stage would have undergone their final cell division only 1 or $2 \mathrm{~d}$ before this time (Banker and Cowan, 1977; Schlessinger et al., 1978). We failed to observe significant expression of MAP2 in non-neuronal cells at any stage of development in culture, even as early as $1 \mathrm{~d}$ after plating. These observations show that the expression of MAP2 occurs quite early in neuronal development and could be among the early events associated with a cell's commitment to the neuronal lineage. It would be of considerable interest to determine the localization of MAPs in the embryonic brain at comparably early stages, during the period of neuronal proliferation, migration, and axon initiation.

Presumably, the molecule we have localized at early stages of development in culture is MAP2b, the more rapidly migrating of the two MAP2 isotypes found in mature brain. Biochemical studies show that MAP2a does not normally appear in the cerebrum until rather late in development, beginning after the second postnatal week (Binder et al., 1984a). It would be of interest to know if MAP2a is expressed as the cells mature in culture and if its expression is influenced by cell-cell interactions. 
It is also significant that MAP2 is preferentially expressed by neurons and becomes preferentially localized to neuronal dendrites in dissociated cell culture, where cell-cell interactions are greatly disrupted. When cells are removed from the embryo at this early stage of development, the subsequent localization of MAP2 must largely occur under endogenous control. It clearly does not depend on contact with other neurons or with glial cells (Cáceres et al., 1984a). The hippocampal neurons in these cultures are in metabolic communication with glial cells, grown on a separate substrate as a source of trophic survival factors (Banker, 1980), but such factors are unlikely to play more than a permissive role in the establishment of cytoskeletal compartmentalization. Taken together with previous observations, which show that polyribosomes also become restricted to the somatodendritic domain in the absence of cell-cell contact (Bartlett and Banker, 1984a), these results indicate that the establishment of neuronal polarity in culture can occur independently of cellcell contact. In this regard neurons are quite different from epithelial cells, which become polarized only under culture conditions that permit the establishment of intercellular junctions (Imhof et al., 1983; Ojakian and Herzlinger, 1984; Sabatini et al., 1983). We do not yet know what role cell interactions that occur in situ, before dissociation, may play in the establishment (or reestablishment) of neuronal polarity in culture.

\section{Localization of MAP2 at early stages of neuronal development}

Without doubt the most unexpected finding in this study was the presence of MAP2 in axons during the first few days of their development in culture. Bernhardt and Matus (1982), based on their studies of Purkinje cell development, had made the attractive suggestion that the differential distribution of HMWMAPs early in neuronal development determined which of a cell's processes subsequently became dendrites and which the axon. Our results show quite the opposite. At the outset of development, MAP2 is present in all of the neuronal processes, axons as well as dendrites. After $1 \mathrm{~d}$ in culture, at a time when MAP2 appears to be uniformly distributed throughout both axons and dendrites, the two classes of processes already differ in their growth characteristics, dendrites elongating about $5 \times$ more slowly than the axons (Banker, unpublished observations).

Only after 3 or $4 \mathrm{~d}$ in culture, when axons and dendrites are already quite distinct morphologically, does the loss of axonal MAP2 begin. The loss in MAP2 staining appears to occur in a proximal-to-distal direction, with axonal staining persisting for the longest time in the region of the growth cone. It is not known if the MAP2 present in developing axons plays a significant functional role. It could be involved in mediating interactions between microtubules and other cytoskeletal elements such as intermediate filaments (LeTerrier et al., 1982; Papasozomenos et al., 1985). Although hippocampal neurons do not contain neurofilaments detectable by immunocytochemistry during the first few days in culture, many of the cells contain vimentin filaments, which are present in both developing axons and dendrites (Shaw et al., 1985). Vallee et al. (1984) have suggested that the functional role of MAP2 at early stages of neuronal development may relate to its interaction with vimentin filaments. Vimentin filaments largely disappear from hippocampal neurons during the first week in culture, which roughly parallels the period when MAP2 becomes restricted to dendrites.

It is not yet known if axons transiently contain MAP2 during normal development in situ. Extrapolation from our results in culture would suggest that significant MAP2 immunoreactivity in the axons of hippocampal neurons would largely disappear by the time of birth, and to date no immunocytochemical studies have examined the localization of MAPs at prenatal stages. At later stages of development, MAP2 is preferentially localized to

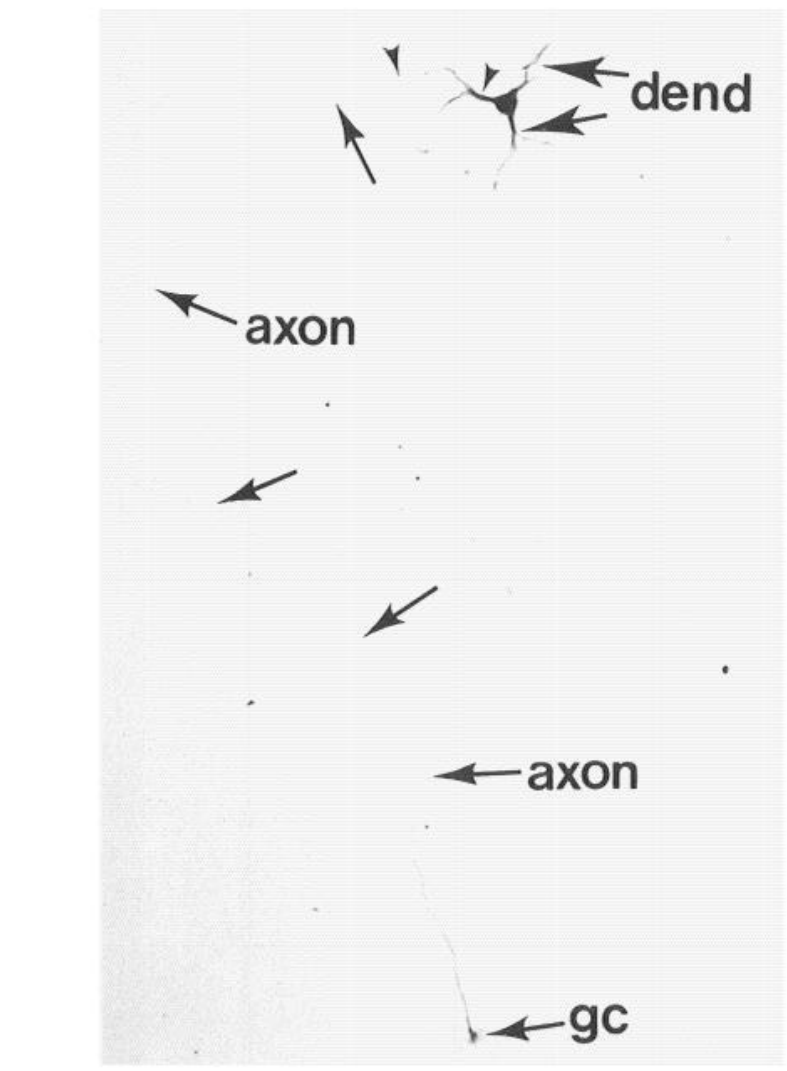

Figure 6. Distribution of MAP2 in a hippocampal neuron after $3 \mathrm{~d}$ in culture. Staining is present in the dendrites (dend), at the base of the axon (arrowheads), and in the region just proximal to the axonal growth cone $(g c)$. The intervening region of the axon (demarcated by the arrows) does not contain detectable MAP2. This culture was fixed in formaldehyde-glutaraldehyde, then reacted with clone AP9. Bar, $50 \mu \mathrm{m} ; \times 200$.

dendrites, but there appears to be more staining of axons than is seen in the mature brain (Frankfurter et al., 1983).

It is also possible that the MAP2 staining of developing axons we observed is an artifact that only occurs in culture. The disassembly of axonal and dendritic microtubules, which is likely to occur when cells are dissociated, might interfere with MAP2 localization when new processes arise. This would be likely if the selective transport of MAP2 into dendrites is linked directly with its biosynthesis, a possibility suggested by Lasek and Brady (1981). In any event, whether or not developing axons contain MAP2 in situ, our results show quite clearly that the presence of MAP2 in a developing neuronal process is not sufficient to determine that it will differentiate as a dendrite.

\section{Localization of tubulin at early stages of dendritic development}

Bernhardt and Matus (1982) were unable to detect immunoreactive tubulin in the dendrites of Purkinje cells at early stages of development, when antibodies directed against the HMWMAPs showed intense staining. From this they concluded that MAPs must play a role in dendritic development that is independent of their ability to associate with microtubules. Other workers have since demonstrated the presence of tubulin in Purkinje cell dendrites at these stages of development by immunocytochemistry, using several different monoclonal antibodies (Burgoyne and Cumming, 1984; Frankfurter et al., 1983), and microtubules have been observed by electron microscopy (Altman, 1972; Frankfurter et al., 1983), although they are few in number. In this regard, Purkinje cells appear to be excep- 
tional, since microtubules are a prominent element in the developing dendrites of nearly all other types of neurons that have been studied (Miller and Peters, 1981; Peters and Feldman, 1973). They are found along the full length of the dendrites, extending to the base of the dendritic growth cones (Hinds and Hinds, 1972; Skoff and Hamburger, 1974; Vaughn et al., 1974).

We were able to detect tubulin by immunocytochemistry in all of the processes of developing hippocampal neurons, including dendrites at their earliest stage of development. At all stages of dendritic development, tubulin immunoreactivity was retained following detergent extraction, suggesting the presence of assembled microtubules. Electron microscopy of hippocampal cells in culture has also revealed densely packed microtubules at all stages of dendritic development, extending to the base of the dendritic growth cone (Bartlett and Banker, 1984a, and unpublished observations). Thus, from the evidence at hand, there is no reason to suppose that the MAP2, which is bound to the cytoskeleton in the developing dendrites of hippocampal neurons, is not associated with microtubules. This does not preclude the possibility that MAP2 is associated with other cytoskeletal elements at special sites, such as dendritic filopodia or growth cones.

\section{Organization of microtubules in hippocampal neurons in culture}

In hippocampal neurons in culture it is most common for the axon to arise as a branch from the proximal portion of a dendrite rather than directly from the cell soma (Bartlett and Banker, 1984a). This also occurs in situ, although it is considerably less common. Since the microtubules in axons and dendrites are different, the two processes arising at such a branch point must contain molecularly distinct populations of microtubules. This is just what we have observed (see Fig. 6): the parent segment and the branch that maintains a dendritic character contain MAP2, while the other, axonal, branch does not. This organization poses intriguing questions concerning the development of axonal and dendritic domains and the selective transport of materials within the cell. Even if axonal and dendritic microtubules arise from two different somatic organizing centers, as has been proposed (Lasek and Brady, 1981), some additional mechanism would still be required to segregate the two classes of microtubules at such a branch point and to direct the transport of materials produced in the cell body into one or the other branch. Thus, the sorting and transport of materials that occur in neurons must be considerably more sophisticated than that in other polarized cells (e.g., Matlin and Simons, 1984).

An analogous situation occurs in dorsal root ganglion cells, where there are differences in the structure and transport of cytoskeletal elements in the peripheral and central branches of the axon (Mori et al., 1979; Smith, 1973; Wujek and Lasek, 1983; Zenker et al., 1975). It has been suggested, although not yet established, that the proximal segment of the axon of dorsal root neurons contains two molecularly distinct populations of microtubules that arc spatially segregated from onc another and that extend into different branches of the axon (Lasek, 1981). This is not implausible, since, during development, the two branches of the axon originally extend from opposite poles of the cell, becoming connected by an intervening axonal segment as the cell body migrates to one side. If, in hippocampal neurons, the processes that give rise to both axonal and dendritic branches contain two spatially segregated microtubule populations, one containing and one lacking MAP2, this should be readily detectable by electron-microscopic immunocytochemistry.

\section{References}

Altman, J. (1972) Postnatal development of the cerebellar cortex in the rat. II. Phases of the maturation of Purkinje cells and the molecular layer. J. Comp. Neurol. 145: 399-464.
Banker, G. A. (1980) Trophic interactions between astroglial cells and hippocampal neurons in culture. Science 209: 809-810.

Banker, G. A., and W. M. Cowan (1977) Rat hippocampal neurons in dispersed cell culture. Brain Res. 126: 397-425.

Banker, G. A., and W. M. Cowan (1979) Further observations on hippocampal neurons in dispersed cell culture. J. Comp. Neurol. 187: 469-494.

Bartlett, W. P., and G. A. Banker (1984a) An electron microscopic study of the development of axons and dendrites by hippocampal neurons in culture. I. Cells which develop without intercellular contacts. J. Neurosci. 4: 1944-1953.

Bartlett, W. P., and G. A. Banker (1984b) An electron microscopic study of the development of axons and dendrites by hippocampal neurons in culture. II. Synaptic relationships. J. Neurosci. 4: 19541965.

Bernardt, R., and A. Matus (1982) Initial phase of dendritic growth: evidence for the involvement of high molecular weight microtubuleassociated proteins (HMWP) before the appearance of tubulin. J. Cell Biol. 92: 589-593.

Binder, L. I., A. Frankfurter, H. Kim, A. Cáceres, M. Payne, and L. I. Rebhun (1984a) Heterogeneity of microtubule-associated protein 2 during rat brain development. Proc. Natl. Acad. Sci. USA 81: 56135617 .

Binder, L. I., A. Frankfurter, and L. I. Rebhun (1984b) A monoclonal antibody to tau-factor localizes predominantly in axons. J. Cell Biol. 99: 191 a.

Bloom, G. S., T. A. Schoenfeld, and R. B. Vallee (1984) Widespread distribution of the major polypeptide component of MAP1 (Microtubule Associated Protein 1) in the nervous system. J. Cell Biol. 98: 320-330.

Booher, J., and M. Sensenbrenner (1972) Growth and cultivation of dissociated neurons and glial cells from embryonic chick, rat, and human brain in flask cultures. Neurobiology 2: 97-105.

Bottenstein, J. E., and G. H. Sato (1979) Growth of a rat neuroblastoma cell line in serum-free supplemented medium. Proc. Natl. Acad. Sci. USA 76: 514-519.

Burgoyne, R. D., and R. Cumming (1984) Ontogeny of microtubuleassociated protein 2 in rat cerebellum: Differential expression of the doublet polypeptides. Neuroscience $11:$ 157-167.

Cáceres, A., L. I. Binder, M. R. Payne, P. Bender, L. Rebhun, and O. Steward (1983) Immunocytochemical localization of actin and microtubule associated protein MAP2 in dendritic spines. Proc. Natl. Acad. Sci. USA 80: 1738-1742.

Cáceres, A., G. Banker, O. Steward, L. Binder, and M. Payne (1984a) MAP2 is localized to the dendrites of hippocampal neurons which develop in culture. Dev. Brain Res. 13: 314-318.

Cáceres, A., L. I. Binder, M. R. Payne, P. Bender, L. Rebhun, and O. Steward (1984b) Differential subcellular localization of tubulin and the microtubule associated protein MAP 2 in brain tissue as revealed by immunocytochemistry with monoclonal hybridoma antibodies. J. Neurosci. 4: 394-410.

Cumming, R., R. D. Burgoyne, and N. A. Lytton (1983) Axonal subpopulations in the central nervous system demonstrated using monoclonal antibodies against alpha-tubulin. Eur. J. Cell Biol. 31: 241248.

DeCamilli, P., P. Miller, F. Navone, W. E. Theurkauf, and R. B. Vallee (1984) Distribution of microtubule associated protein 2 (MAP2) in the nervous system of the rat studied by immunoflourescence. Neuroscience 11: 819-846.

Francon, J., A. Lennon, A. Fellous, A. Mareck, M. Pierre, and J. Nunez (1982) Heterogeneity of microtubule-associated proteins in brain development. Eur. J. Biochem. 129: 465-471.

Frankfurter, A., A. Cáceres, L. I. Binder, and L. I. Rebhun (1983) Immunohistochemical localization of tubulin and the high molecular weight microtubule-associated proteins (MAP1 and MAP2) in the developing cerebellum of the rat. Soc. Neurosci. Abstr. 9: 851.

Goldstein, M. E., L. A. Sternberger, and N. H. Sternberger (1983) Microheterogeneity ("neurotypy") of neurofilament proteins. Proc. Natl. Acad. Sci. USA 80: 3101-3105.

Gozes, I., and C. J. Barnstable (1982) Monoclonal antibodies that recognize discrete forms of tubulin. Proc. Natl. Acad. Sci. USA 79: 2579-2583.

Gozes, I., and K. J. Sweadner (1981) Multiple tubulin forms are expressed by a single neuron. Nature $294: 477-479$. 
Greene, L. A., R. K. H. Liem, and M. L. Shelanski (1983) Regulation of a high molecular weight microtubule-associated protein in $\mathrm{PC} 12$ cells by Nerve Growth Factor. J. Cell Biol. 96: 76-83.

Hillman, D. E. (1979) Neuronal shape parameters and substructures as a basis of neuronal form. In The Neurosciences: Fourth Study Program, F. O. Schmidt and F. W. Worden, eds., pp. 477-498, MIT Press, Cambridge, MA.

Hinds, J. W., and P. L. Hinds (1972) Reconstruction of dendritic growth cones in neonatal mouse olfactory bulb. J. Neurocytol. 1: 169187.

Hirokawa, N., M. A. Glicksman, and M. B. Willard (1984) Organization of mammalian neurofilament polypeptides within the neuronal cytoskeleton. J. Cell Biol. 98: 1523-1536.

Huber, G., and A. Matus (1984a) Differences in the cellular distribution of two microtubule-associated proteins, MAP1 and MAP2, in rat braill. J. Neurosci. 4: 151-160.

Huber, G., and A. Matus (1984b) Immunocytochemical localization of microtubule-associated protein 1 in rat cerebellum using monoclonal antibodies. J. Cell Biol. 98: 777-781.

Imhof, B. A., H. P. Vollmers, S. L. Goodman, and W. Birchmeier (1983) Cell-cell interaction and polarity of epithelial cells; Specific perturbation using a monoclonal antibody. Cell 35: 667-675.

Izant, J. G., and J. R. McIntosh (1980) Microtubule associated proteins: A monoclonal antibody to MAP2 binds to differentiated neurons. Proc. Natl. Acad. Sci. USA 77: 4747-4754.

Lasek, R. J. (1981) The dynamic ordering of neuronal cytoskeletons. Neurosci. Res. Program Bull. 19: 7-32.

Lasek, R. J. (1982) Translocation of the neuronal cytoskeleton and axonal locomotion. Philos. Trans. R. Soc. London [Biol.] 299: 313 327.

Lasek, R. J., and S. T. Brady (1981) The axon: A prototype for studying expressional cytoplasm. Cold Spring Harbor Symp. Quant. Biol. 46. $113-124$.

Lasek, R. J., M. M. Oblinger, and P. F. Drake (1983) Molecular biology of neuronal geometry: Expression of neurofilament genes influences axonal diameter. Cold Spring Harbor Symp. Quant. Biol. 48: 731744.

LeTerrier, J. F., R. K. H. I eim, and M. Shelanski (1982) Interactions between neurofilament and microtubule-associated proteins: A possible mechanism for intra-organelle bridging. J. Cell Biol. 95: 982986

Mareck, A., A. Fellous, J. Francon, and J. Nunez (1980) Changes in composition and activity of microtubule-associated proteins during brain development. Nature 284: 353-355.

Matlin, K. S., and K. Simons (1984) Sorting of an apical plasma membrane glycoprotein occurs before it reaches the cell surface in cultured epithelial cells. J. Cell Biol. 99: 2131-2139.

Matus, A., R. Bernhardt, and T. Hugh-Jones (1981) High molecular weight microtubule associated proteins are preferentially associated with dendritic microtubules in brain. Proc. Natl. Acad. Sci. USA 78: 3010-3014.

Miller, M., and A. Peters (1981) Maturation of rat visual cortex. II A combined Golgi-electron microscopy study of pyramidal neurons. J. Comp. Neurol. 203: 555-573.

Mori, H., Y. Komiya, and M. Kurokawa (1979) Slowly migrating axonal polypeptides: Inequalities in their rate and amount of transport between two branches of bifurcating axons. J. Cell Biol. 82: 174-184.

Moura Neto, V., M. Mallat, C. Jeantet, and A. Prochiantz (1983) Microheterogeneity of tubulin proteins in neuronal and glial cells from the mouse brain in culture. EMBO J. 2: 1243-1248.

Ojakian, G. K., and D. A. Herzlinger (1984) Analysis of epithelial cell surface polarity with monoclonal antibodies. Fed. Proc. 43: 22082216.

Olmstead, J. B., and H. D. Lyon (1981) A microtubule-associated protein specific to differentiated neuroblastoma cells. J. Biol. Chem. 256: $3507-3511$
Pachter, J. S., and R. K. H. Liem (1984) The differential appearance of neurofilament triplet polypeptides in the developing rat optic nerve. Dev. Biol. 103: 200-210.

Papasozomenos, S. C., L. I. Binder, P. Bender, and M. R. Payne (1985) Microtubule-associated protein 2 (MAP2) within axons of spinal motor neurons: Associations with microtubules and neurofilaments in normal and beta, beta'-iminodiproprionitrile-treated axons. J. Cell Biol 100: 74-85.

Payne, M. R. (1982) Monoclonal antibodies to the contractile proteins. In Cell and Muscle Motility, Vol. 4, R. Dowben and J. Shay, eds., pp. 137-177, Plenum, New York.

Peters, A., and M. Feldman (1973) The cortical plate and molecular layer of the late rat fetus. Z. Anat. Entwickl. Gesch. 141: 3-37.

Peters, A., and J. E. Vaughn (1967) Microtubules and filaments in the axons and astrocytes of early postnatal rat optic nerves. J. Cell Biol. 32: 113-119.

Raff, M. C., E. R. Abney, J. Cohen, R. Lindsay, and M. Noble (1983) Two types of astrocytes in cultures of developing rat white matter: Differences in morphology, surface gangliosides, and growth characteristics. J. Neurosci. 3: 1289-1300.

Sabatini, D. D., E. B. Griepp, E. J. Rodriguez-Boulan, W. J. Dolan, E. S. Robbins, S. Papadopoulos, I. E. Ivanov, and M. J. Rindler (1983) Biogenesis of epithelial cell polarity. Mod. Cell Biol. 2: 419-450.

Schlessinger, A. R., W. M. Cowan, and L. W. Swanson (1978) The time of origin of neurons in Ammon's horn and the associated retrohippocampal fields. Anat. Embryol. 154: 153-173.

Schliwa, M., and J. VanBlerkom (1981) Structural interactions of cytoskeletal elements. J. Cell Biol. 90: 222-235.

Shaw, G., G. A. Banker, and K. Weber (in press) An immunofluorescence study of neurofilament protein expression by developing hippocampal neurons in tissue culture. Eur. J. Cell Biol.

Shaw, G., M. Osborn, and K. Weber (1981) An immunofluorescence microscopical study of the neurofilament triplet proteins, vimentin, and the glial fibrillary acidic protein within the adult rat brain. Eur. J. Cell Biol. 26: 68-82.

Skoff, R. P., and V. Hamburger (1974) Fine structure of dendritic and axonal growth cones in embryonic chick spinal cord. J. Comp. Neurol. 153: $107-148$.

Smith, R. S. (1973) Microtubule and neurofilament densities in amphibian spinal root fibers: Relationships to axoplasmic transport. Can. J. Physiol. Pharmacol. 51: 798-806.

Solomon, F. (1981) Specification of cell morphology by endogenous determinants. J. Cell Biol. 90: 547-553.

Solomon, F., and A. Zurn (1981) The cytoskeleton and specification of neuronal morphology. Neurosci. Res. Program Bull. 19: 100-107.

Tsui, H. T., H. Ris, and W. L. Klein (1983) Ultrastructural networks in growth cones and neurites of cultured nervous system neurons. Proc. Natl. Acad. Sci. USA 80: 5779-5783.

Tytell, M., S. T. Brady, and R. J. Lasek (1984) Axonal transport of a subclass of tau proteins: Evidence for the regional differentiation of microtubules in neurons. Proc. Natl. Acad. Sci. USA 81: 1570-1574.

Vallee, R. (1982) A taxol dependent procedure for the isolation of microtubules and microtubule associated proteins (MAPs). J. Cell Biol. 92: 435-442.

Vallee, R. B., G. S. Bloom, and W. E. Theurkauf (1984) Microtubuleassociated proteins: Subunits of the cytomatrix. J. Cell Biol. 99: 38s$44 \mathrm{~s}$

Vaughn, J. E., C. K. Henrikson, and J. A. Grieshaber (1974) A quantitative study of synapses on motor neuron dendritic growth cones in developing mouse spinal cord. J. Cell Biol. 60: 6664-6672.

Wujek, J. R., and R. J. Lasek (1983) Correlation of axonal regeneration and slow component $\mathrm{b}$ in two branches of a single axon. J. Neurosci. 3: 243-251.

Zenker, W., R. Mayr, and H. Gruber (1975) Neurotubules: Different densities in peripheral motor and sensory nerve fibers. Experientia 31: $318-320$. 